\title{
Metastatic site discriminates survival benefit of primary surgery for differentiated thyroid cancer with distant metastases: A real-world observational study
}

WU DING ( $\nabla$ wuding0320@163.com )

Shaoxing Second hospital

Guodong Ruan

Shaoxing Second Hospital

Jianming zhu

Shaoxing Second Hospital

Chuanjian Tu

Shaoxing University Affiliated Hospital

Zhian Li

Shaoxing Second Hospital

\section{Research}

Keywords: Differentiated thyroid cancer, Primary surgery, Metastatic site, Survival

Posted Date: March 26th, 2020

DOI: https://doi.org/10.21203/rs.3.rs-19233/v1

License: (c) (i) This work is licensed under a Creative Commons Attribution 4.0 International License.

Read Full License 


\section{Abstract}

Background: Role of surgery in the management of differentiated thyroid cancer (DTC) with distant metastases (DM) remains controversial. We aimed to determine the survival benefit of primary surgery on the basis of metastatic site.

Methods: A retrospective cohort study based on the SEER database was conducted to identify DTC patients with DM diagnosed between 2010 and 2016. Patients were divided into surgery and non-surgery group, and propensity score weighting was used to balance clinicopathologic factors between groups.

Results: Of 3537 DTC patients with DM, 956 (66.0\%) cases were managed with surgery and 493 (34.0\%) cases were managed without surgery. There were 798 all-cause deaths and 704 DTC specific deaths over a median follow-up of 22 months. The weighted 3-year overall survival (OS) for the surgery group was $55.2 \%$, compared to $27.8 \%(P<0.001)$ for the non-surgery group. The magnitude of the survival difference with surgery was significantly correlated with metastatic sites (Pinteraction<0.001). Significant survival improvements in surgery group compared with non-surgery group were observed in patients with lung-only metastasis (adjusted HR $=0.45, \mathrm{P}<0.001$ ), bone-only metastasis (adjusted $\mathrm{HR}=0.40, \mathrm{P}<$ 0.001 ), or liver-only metastasis (adjusted $H R=0.27, P<0.001$ ), whereas survival no improvement of surgery was found for patients with brain-only metastasis (adjusted $H R=0.57, P=0.059$ ) or multiply organs distant metastases (adjusted HR $=0.81, \mathrm{P}=0.099$ ).

Conclusion: The survival benefit offered by surgery for DTC patients with DM varies by metastatic sites. Decisions for primary surgery of DTC patients with DM should be tailored according to metastatic sites.

\section{Background}

Differentiated thyroid carcinoma (DTC), which includes papillary thyroid carcinoma (PTC) and follicular thyroid carcinoma (FTC), is one of the most curable endocrine cancers. In the majority of patients with DTC, the cause of death is due to distant metastases (DM) rather than locoregional recurrence. Between $1 \%$ and $4 \%$ of DTC patients presented with distant disease at the time of initial diagnosis $(1,2)$, and the 10-year cancer-specific survival of patients with metastatic DTC up to $90 \%(3-8)$.

DTC with DM at initial diagnosis (primary DM) had markedly varying clinical outcomes from rapid progression and death to complete remission (4-10), the difference of metastatic disease site was considered as a possible reason for inconsistent outcome. The site of metastasis had been reported as significant association between extrapulmonary metastases and poor prognosis $(4,7-8)$. Of note, the survival benefit of the removal of the primary tumor in patients with primary DM among those trials is controversial.

The aim of this real-world observational study was to determine the survival benefit of primary surgery among patient subpopulations stratified by metastatic sites who presented with DM at initial diagnosis. 
We hypothesized that the local surgery may confer a survival benefit to patients with low metastatic tumor burden.

\section{Methods}

\section{Study Design and Data Source}

After receiving an exemption from the Partners HealthCare Institutional Review Board, we performed a retrospective longitudinal cohort study using data obtained from the SEER Program of the National Cancer Institute.

The Surveillance, Epidemiology, and End Results (SEER) database (http://seer.cancer.gov/) sponsored by the National Cancer Institute $(\mathrm{NCl})$ covered 18 population-based registries, involving a large proportion (28\%) of US people. We used the November 2018 SEER-18 submission for this retrospective longitudinal cohort study, which included patients from geographic regions covered as follows: Metropolitan Atlanta, Connecticut, Detroit, Hawaii, lowa, New Mexico, San Francisco-Oakland, Seattle-Puget Sound, Los Angeles, San Jose-Monterey, Utah, Rural and Greater Georgia, Alaska, Greater California, Kentucky, Louisiana, and New Jersey. We identified 3537 patients who were first initially diagnosed as DTC with DM between January 1, 2010, and December 31, 2016 (Fig. 1). We excluded patients only diagnosed by autopsy or death certifications, moreover, those cases without histological confirm were also excluded. Patient receiving surgery for metastatic sits or unknown sites as well as cases with unknown metastatic sites were excluded. At last, eligible 1449 DTC patients with primary DM were included in this study, who were grouped according to whether they underwent primary tumor surgery $(N=956)$ or not $(N=493)$. Considering this study was reviewed and approved by the institutional review board at the Shaoxing Second hospital and determined to not be human participant research, patient consent was not involved.

\section{Identification of key variables}

The SEER*Stat software (version 8.3.6) was used to extract relevant information, including patient identification, age of diagnosis, year of diagnosis, sex, tumor size, regional lymph node status, race/ethnicity, marital status, distant metastatic site, histology type, nuclear grade, surgery, chemotherapy, radiation therapy, cause-specific death classification, other cause of death classification and survival month.

International Classification of Diseases for Oncology (ICD-0-3) (13)was used to identify the cancer site and histology type, and cases of papillary and follicular thyroid cancer were selected using the restrictions and . We placed Hurthle cell carcinoma $(I C D O-3=8290)$ into the category of follicular carcinomas, as used in Lim et al. $(14,15)$. To investigate the benefit of primary surgery on the basis of metastasis sites, the variable was categorized into single organ and multiple organs metastases. The single organ metastasis was further classified into bone-only, liver-only, lung-only and brain-only 
metastasis, and multiple organs metastases were classified into multiply organs metastases including brain or excluding brain.

\section{Main Outcome Measure}

The primary endpoint of this study was overall survival (OS) and disease-specific survival (DSS), which was defined as an internal from time of diagnosis to overall death (DTC specific death) or date of last contact and considered as censored statuses if patients were alive until date of last contact. SEER defines mortality data based on the International Classification of Diseases Revisions 8 to 10, which categorized the cause of death as DTC specific death and other cause death.

\section{Statistical Analysis}

For this study, we adopt the similar statistical analytic approaches with previous studies $(16,17)$ that examined the benefit of interventions for breast cancer subsets. Clinicopathologic factors were compared between the surgery groups and non-surgery groups using Pearson $\chi 2$ tests. Multiple imputation of missing data was performed by a multivariate logistic regression model, and 10 cycles were repeated to produce a final data set. Imputation model included these variables as follows: race (white, black, or others), marital status (single, separated and married), nuclear grade (I,II,IIIIIV), tumor size classification $(0-2 \mathrm{~cm}, 2-4 \mathrm{~cm}, \mathrm{or} \otimes 4 \mathrm{~cm})$, and regional lymph node status(positive or negative).

Propensity score weighting was then used to balance patient characteristics between the surgery and non-surgery groups (18.19). we calculated the propensity scores based on patient age, year of diagnosis, race, sex, tumor size, regional lymph node status, marital status, distant metastatic site, histology type, nuclear grade, chemotherapy, radiation therapy through a logistic regression model for receipt of surgery. From the model, the inverse predicted probability of breast surgery assignment was used to define weights for patients who received surgery( 1 /probability) and for those who did not receive surgery $(1 /[1$ probability]). Patient characteristics after propensity score adjustment are shown to be balanced in Table 2.

The hazard ratios for the DSS and OS of patients in the surgery group compared with patients in the nonsurgery group were evaluated using propensity score weights for log-rank tests and Cox regression models. Hazard ratios (HRs) of OS and DSS were reported from multivariable models that adjusted for patient age, year of diagnosis, race, sex, tumor size, regional lymph node status, marital status, distant metastatic sites, histology type, nuclear grade, chemotherapy, radiation therapy. Similar procedures were also performed among subgroups defined by metastatic sites, and interaction tests were conducted using a likelihood ratio test to explore whether any survival benefit conferred by surgery varied across subgroups.

In addition, to assess the stability of our results, we conducted a series of sensitivity analyses. First, the entire analyses were repeated after imputation unknown data using random survival 
forest methodology. Then, proportional subdistribution hazards model was used to calculated HR of OS and DSS between surgery and non-surgery group after adjusting competing events (20) such as death from other causes. Second, we performed the analysis after restriction to patients in the SEER 9 registry, because the data in the SEER 9 registry are more accurate than the data in newer SEER registries (21). Last, since age under 55 years was a good prognosis factor whether there is distant metastasis, we excluded the patients with primary DM under the age of 55 years who often sought surgery for longer survival.

All $P$ values were calculated from 2-sided tests with threshold of 0.05 to evaluate statistical significance of survival benefit by surgery, and all statistical analyses were performed using R software (version 3.6.1).

\section{Results}

\section{Patient characteristics}

We identified 3537 eligible DTC patients with DM at the time of initial treatment on the basis of our inclusion and exclusion criteria (Fig. 1). Of this cohort, 956 (66.0\%) received the primary surgery, and 493 (34.0\%) patients were stratified into non-surgery group. Clinicopathologic factors and SEER cancer registries according to receipt of primary surgery were listed in Table 1. The final data after multiple imputations was exhibited in Table 2. Balance in patient characteristics was achieved after propensity score adjustments for estimating average treatment effect, as shown in Table 2. The proportion of patient with age under 55 years, earlier year of diagnosis, white/ethnicity, male, follicular, small tumor size, regional node negative, and nuclear grade was larger for the surgery group compared with the nonsurgery group. 
Table 1

Patient characteristics by receipt of primary surgery.

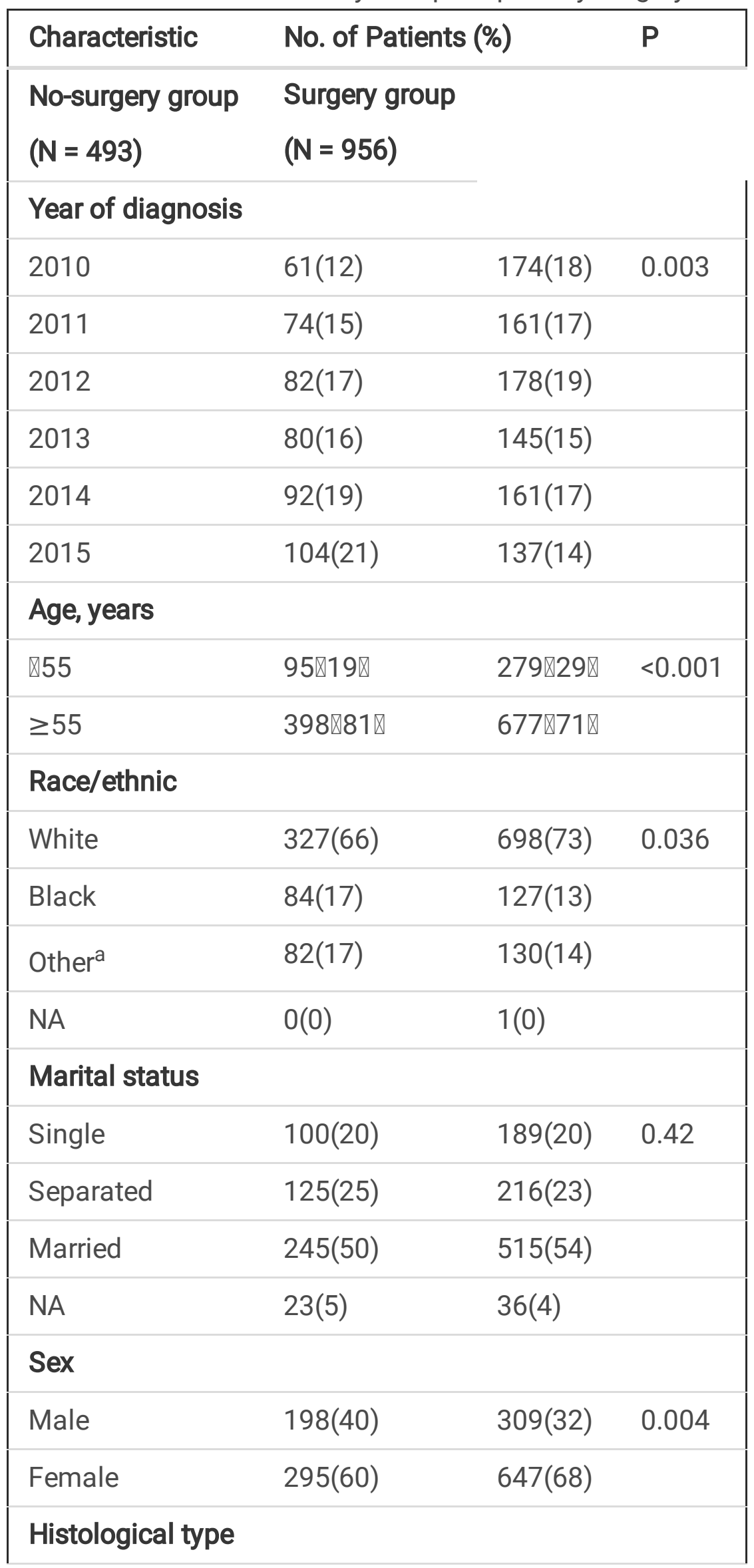




\begin{tabular}{|c|c|c|c|}
\hline \multirow{2}{*}{$\begin{array}{l}\text { Characteristic } \\
\text { Papillary }\end{array}$} & \multicolumn{2}{|c|}{ No. of Patients (\%) } & \multirow{2}{*}{$\begin{array}{l}\mathbf{P} \\
0.042\end{array}$} \\
\hline & $449(91)$ & $835(87)$ & \\
\hline Follicular & $44(9)$ & $121(13)$ & \\
\hline \multicolumn{4}{|l|}{ Grade } \\
\hline 1 & $25(5)$ & $92(10)$ & $<0.001$ \\
\hline II & $36(7)$ & $100(10)$ & \\
\hline III & $39(8)$ & $237(25)$ & \\
\hline IV & $22(4)$ & $126(13)$ & \\
\hline NA & $371(75)$ & $401(42)$ & \\
\hline \multicolumn{4}{|c|}{ Tumor size (cm) } \\
\hline$\leq 2$ & $44(9)$ & 154(16) & $<0.001$ \\
\hline $2-4$ & $90(18)$ & 197(21) & \\
\hline$\nabla 4$ & $173(35)$ & $471(49)$ & \\
\hline NA & 186(38) & 134(14) & \\
\hline \multicolumn{4}{|c|}{ Regional node positive } \\
\hline No & 168(34) & $447(47)$ & $<0.001$ \\
\hline Yes & $250(51)$ & $452(47)$ & \\
\hline NA & $75(15)$ & $57(6)$ & \\
\hline \multicolumn{4}{|l|}{ Radiation } \\
\hline No & $300(61)$ & $511(53)$ & $<0.001$ \\
\hline RAl & $10(2)$ & $287(30)$ & \\
\hline EBRT & 183(37) & $158(17)$ & \\
\hline \multicolumn{4}{|l|}{ Chemotherapy } \\
\hline No & 172(35) & $512(54)$ & $<0.001$ \\
\hline Yes & $321(65)$ & $444(46)$ & \\
\hline \multicolumn{4}{|c|}{ Distant metastatic site } \\
\hline \multicolumn{4}{|l|}{ Bone } \\
\hline No & $311(63)$ & $703(74)$ & $<0.001$ \\
\hline Yes & 182(37) & $253(26)$ & \\
\hline
\end{tabular}




\begin{tabular}{|llll|}
\hline Characteristic & No. of Patients (\%) & P \\
\hline Brain & & & \\
\hline No & $386(78)$ & $925(97)$ & $<0.001$ \\
\hline Yes & $107(22)$ & $31(3)$ & \\
\hline Liver & & & \\
\hline No & $390(79)$ & $719(75)$ & 0.111 \\
\hline Yes & $103(21)$ & $237(25)$ & \\
\hline Lung & & & \\
\hline No & $228(46)$ & $387(40)$ & 0.041 \\
Yes & $265(54)$ & $569(60)$ & \\
\hline
\end{tabular}

Abbreviations: NA, not available; RAl: radioactive iodine; EBRT: external beam radiation therapy. a American Indian/AK Native, Asian/Pacific Islander. 
Table 2

Patient characteristics by weighted by propensity score.

\begin{tabular}{|c|c|c|}
\hline \multirow[t]{2}{*}{ Characteristic } & \multicolumn{2}{|l|}{ No. of Patients (\%) } \\
\hline & $\begin{array}{l}\text { No-surgery group } \\
(\mathrm{N}=493)\end{array}$ & $\begin{array}{l}\text { Surgery group } \\
(\mathrm{N}=956)\end{array}$ \\
\hline \multicolumn{3}{|c|}{ Year of diagnosis } \\
\hline 2010 & $68(13.8)$ & $156(16.3)$ \\
\hline 2011 & $85(17.2)$ & $143(15.0)$ \\
\hline 2012 & $82(16.6)$ & 169(17.7) \\
\hline 2013 & $88(17.9)$ & $161(16.8)$ \\
\hline 2014 & $78(15.8)$ & 169(17.7) \\
\hline 2015 & $92(18.7)$ & $160(16.7)$ \\
\hline \multicolumn{3}{|l|}{ Age, years } \\
\hline$\otimes 55$ & $148(30.0)$ & $254(26.6)$ \\
\hline$\geq 55$ & $345(70.0)$ & 702(73.4) \\
\hline \multicolumn{3}{|l|}{ Race/ethnic } \\
\hline White & $336 \varangle 68.2 \rrbracket$ & $678 \otimes 70.9 \rrbracket$ \\
\hline Black & $90 \rrbracket 18.2 \rrbracket$ & 131ه13.7区 \\
\hline Other ${ }^{a}$ & $67 \rrbracket 13.6 \rrbracket$ & 147ه15.4区 \\
\hline \multicolumn{3}{|l|}{ Marital status } \\
\hline Single & $245(49.7)$ & $533(55.8)$ \\
\hline Separated & $141(28.5)$ & $180(18.8)$ \\
\hline Married & 107(21.8) & $243(25.4)$ \\
\hline \multicolumn{3}{|l|}{ Sex } \\
\hline Male & 168(34.1) & $351(36.7)$ \\
\hline Female & $325(65.9)$ & $605(63.3)$ \\
\hline \multicolumn{3}{|c|}{ Histological type } \\
\hline Papillary & $424(86.1)$ & $846(88.5)$ \\
\hline Follicular & $69(13.9)$ & $110(11.5)$ \\
\hline
\end{tabular}




\begin{tabular}{|c|c|c|}
\hline 1 & $92(18.6)$ & $105(21.2)$ \\
\hline II & 106(21.5) & $105(21.2)$ \\
\hline III & $211(42.8)$ & $377(39.4)$ \\
\hline IV & $84(17.1)$ & 173(18.2) \\
\hline \multicolumn{3}{|c|}{ Tumor size (cm) } \\
\hline$\leq 2$ & 73囚14.9凶 & 173ه18.1区 \\
\hline $2-4$ & $125 \rrbracket 25.4 \rrbracket$ & $215 \rrbracket 22.5 \rrbracket$ \\
\hline$\llbracket 4$ & 294囚59.7凶 & $568 \rrbracket 59.4 \rrbracket$ \\
\hline \multicolumn{3}{|c|}{ Regional node positive } \\
\hline No & $256 \rrbracket 52.0 \rrbracket$ & $453 \rrbracket 47.4 \rrbracket$ \\
\hline Yes & $237 \rrbracket 48.0 \rrbracket$ & $503 \rrbracket 52.6 \rrbracket$ \\
\hline \multicolumn{3}{|c|}{ Radiation } \\
\hline No & $340(69.0)$ & $513(53.7)$ \\
\hline RAl & 15(3.1) & $248(25.9)$ \\
\hline EBRT & 138(27.9) & 195(20.4) \\
\hline \multicolumn{3}{|c|}{ Chemotherapy } \\
\hline No & $247(50.1)$ & $464(48.5)$ \\
\hline Yes & $246(49.9)$ & 491(51.5) \\
\hline \multicolumn{3}{|c|}{ Bone } \\
\hline No & $349 \bigotimes 70.8 \rrbracket$ & $666(69.7)$ \\
\hline Yes & 144囚29.2》 & $290(30.3)$ \\
\hline \multicolumn{3}{|c|}{ Brain } \\
\hline No & 448(90.9) & $863(90.3)$ \\
\hline Yes & $45(9.1)$ & 93(9.7) \\
\hline \multicolumn{3}{|l|}{ Liver } \\
\hline No & $356(72.3)$ & $727(76.0)$ \\
\hline Yes & $137(27.7)$ & $229(24.0)$ \\
\hline \multicolumn{3}{|l|}{ Lung } \\
\hline No & $222(45.0)$ & $414(42.3)$ \\
\hline
\end{tabular}


Abbreviations: RAl: radioactive iodine; EBRT: external beam radiation therapy.

a American Indian/AK Native, Asian/Pacific Islander.

\section{Sites of distant metastases}

A total of 1747 sites of distant metastases were identified in the 3537 patients of DTC with primary DM. The lung was the most common site of distant metastasis $(834,47.7 \%)$, followed by bone $(435,24.9 \%)$, liver (340, 19.5\%) and brain (138, 7.9\%) (Fig. 2). There are 1208 (83.4\%) patients with single organ metastasis, and 241 (16.6\%) patients with multiple organs metastases (Fig. 2).

\section{Survival benefit of primary surgery}

After a median follow-up time of 22 months from diagnosis (interquartile range, 12-41 months), 704 DTC specific deaths (48.6\%), 94 deaths from other cancer causes (6.5\%). The 3-year OS rate weighted by inverse propensity score was $55.2 \%$ in the surgery group and $27.8 \%$ in the non-surgery group ((log-rank test, $\mathrm{P}<0.001 ; \mathrm{HR}, 0.47 ; 95 \% \mathrm{Cl}, 0.43$ to 0.52 ). The 3-year DSS rate weighted by inverse propensity score was $58.6 \%$ in the surgery group and $34.6 \%$ in the non-surgery group (log-rank test, $P<0.001 ; \mathrm{HR}, 0.50$; $95 \% \mathrm{Cl}, 0.45$ to 0.56 ). Statistically significant difference from the proportional hazard assumption in the Cox regression hazard model adjusting for age, race, marital status, sex, tumor size, regional lymph node status, nuclear grade, histological type, radiation, chemotherapy and metastasis sites $(P<0.001$; adjusted HR for OS, $0.51 ; 95 \% \mathrm{Cl}, 0.46$ to $0.56 ; \mathrm{P}<0.001$; adjusted HR for DSS, $0.54 ; 95 \% \mathrm{Cl}, 0.48$ to 0.60 ).

\section{Survival benefit of surgery according to metastasis sites}

Among 1208 patients with single organ metastasis, there are 627 patients with lung metastasis, 253 patients with bone metastasis, 251 patients with liver metastasis, 77 patients with brain metastasis. The magnitude of improved survival among patients receiving primary surgery was significantly correlated

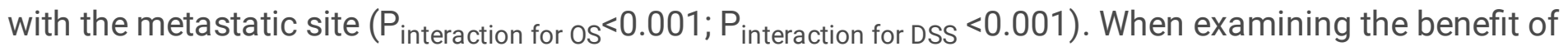
primary surgery stratified by metastatic site, we found that the survival for the primary surgery group was significantly better than that observed in the non-surgery group for patients with lung, bone, or liver metastasis (lung: OS, HR, $0.45 ; 95 \% \mathrm{Cl}, 0.38$ to 0.54 , $\mathrm{P}<0.001$; DSS, $\mathrm{HR}, 0.50 ; 95 \% \mathrm{Cl}, 0.42$ to $0.60 ; \mathrm{P}<$ 0.001; bone: OS, HR, $0.40 ; 95 \% \mathrm{Cl}, 0.29$ to $0.56, \mathrm{P}<0.001$; DSS, HR, $0.35 ; 95 \% \mathrm{Cl}, 0.24$ to $0.50 ; \mathrm{P}<0.001$; liver: OS, HR, 0.27 ; $95 \% \mathrm{Cl}, 0.21$ to $0.36, \mathrm{P}<0.001$; DSS, $\mathrm{HR}, 0.21$; $95 \% \mathrm{Cl}, 0.27$ to 0.36 ; $\mathrm{P}<0.001$; Fig. 2). Whereas patients with brain metastasis demonstrated no significant difference in OS or DSS (OS: HR, $0.57,95 \% \mathrm{Cl}, 0.32$ to $1.02, \mathrm{P}=0.059$; DSS: $\mathrm{HR}, 0.59 ; 95 \% \mathrm{Cl}, 0.33$ to $1.05, \mathrm{P}=0.073$; Fig. 3), similar result were found in patient with multiple organs metastases include brain metastases(OS: $\mathrm{HR}, 0.77,95 \% \mathrm{Cl}$, 0.26 to $2.30, P=0.644$; DSS: HR, $0.69 ; 95 \% \mathrm{Cl}, 0.19$ to $2.45, \mathrm{P}=0.566$; Fig. 3). However, we observed the OS and DSS benefit of primary surgery for DTC patients with multiple organs metastases exclude brain metastasis, although the benefit of DSS had not statistical difference (OS: HR, $0.70,95 \% \mathrm{Cl}, 0.51$ to 0.95 , $P=0.021$; DSS: HR, $0.77,95 \% \mathrm{Cl}, 0.56$ to $1.06, P=0.109$, Fig. 3). 
In sensitivity analyses performed after the exclusion of patients with age under 55 years, after restriction of patients within SEER 9, after repeating analyses using the proportional subdistribution hazards model, we observed similar findings.

\section{Discussion}

Although DTC is a disease with generally a good outcome, patients presenting with distant metastatic disease have less favorable outcomes. Although distant metastasis from DTC are usually slow-growing compared with other malignancies, some of the patients with these conditions die from disease-specific causes. For this reason, many risk stratification algorithms consider such cases to be high risk. Despite the higher chance of poor outcome, current treatment guidelines advocate an aggressive approach to management with surgery and postoperative radioactive iodine (RAI) therapy $(22,23)$. Treatment consists of total thyroidectomy, neck dissection as indicated by the detection of disease in the central and/or lateral neck, followed by RAl therapy in most patients.

The prognostic value of distant metastatic site has been widely studied. In this study, the commonest site of metastases was lung ( $42 \%)$, bone (17\%), liver (17\%), brain (5\%) and then the group with multiple sites affected $(19 \%)$. These results were similar to those of previous reports $(4,11,24)$. A number of studies finding that the prognosis of patients is related to the location of metastases $(4,7-8)$. Patients with brain metastases have a worse outcome compared to other groups. In this study, the 3-year DSS rate of brain metastasis from DTC (28.7\%) was the poorest compared with other organ metastasis (lung metastasis $45.9 \%$, liver metastasis $40.9 \%$, and bone metastasis $50.4 \%$ ).

In this study, we observed higher overall and DTC specific survival in patients with primary DM managed with surgery than individuals without surgery, and this finding based on the real-world study was consistent with prior studies (25). This may be due to a possible heterogeneous treatment effect of primary surgery when metastatic sites varied. Our findings indicated that definitive local surgery of patients with only-brain metastasis offered no significant survival benefit over non-operative management, but a significant survival improvement for surgery was observed in other single organ metastasis DTC patients. For patients with multiply organs distant metastasis, surgery could also improve DTC specific survival in patients without brain metastasis, whereas surgery offered no overall survival or DTC specific survival benefit for patients with brain metastasis. These results suggest that individualized decisions for primary surgery of primary DM patients could be tailored on the basis of metastatic sites. Although there were no widely-accepted guidelines exist on the management of metastatic thyroid carcinoma, patients who underwent surgical resection had significantly longer survival than patients who did not in this study. We thus believe that the presence of DM alone does not automatically exclude the indication of aggressive local radical resection to clear margins. DM is undoubtedly the most common primary cause of cancer death in DTC, but other characteristics of DTC in the high-risk group, such as invasion to surrounding organs or anaplastic transformation in the neck lesion, can also become fatal. Haq et al. reported that lesser surgery (biopsy or nodulectomy) of the primary neoplasm in patients with DM was associated with worse survival compared to radical surgery (11). Although aggressive radical resection sometimes results in substantial perioperative or long-term 
morbidity, such as dysphonia, death from local causes including gradual suffocation from the tracheal involvement, dysphasia, and bleeding, most of them can be prevented.

Surgery, radiotherapy, and RAl therapy have been widely used to treat the DTC with distant metastases. In our study, the benefit of surgical intervention of locally disease was limited in patients with brain metastasis. RAl is an important systemic therapy for patients with brain metastases with RAl-avid disease who respond to this therapy (26). Unfortunately, based on current case reports and retrospective series, RAl uptake by cranial metastatic lesions is quite low, with a reported range from $0-25 \%$ of cases (27-31), possible explanations may be decreased expression of the sodium iodide symporter (NIS) or diminished membrane targeting of NIS in metastatic lesions (32). Furthermore, some studies have suggested that the prognosis in patients who present initially with metastases versus those who subsequently develop metastases may be different $(7,11,12,33)$. The patients presenting initially with metastases appear to have relatively favorable outcomes compared with patients developing metastases after initial treatment. This result will due to the patients with distant metastasis at presentation are 'treatment-naive,' in particular with respect to RAl, and therefore strongly RAI avid (24). One study has found that complete local control and metastatic site RI avidity were independent predictors of an increased DSS or OS relative risk, whereas only complete local control was an independent OS predictor in patients presenting with primary DM (7).

The age at diagnosis of the initial cancer is known to be a valuable prognostic factor for the recurrence and mortality of DTC (34). In the current series multivariate analysis identified age was an independent risk factor for bad prognostic feature. We found that age over 55 years was significant predictors of a poor outcome, the results showed that an improved survival with patients younger than 55 years at presentation compared to those older than 55 years (3-year DSS $52 \%$ vs. $45 \%$, respectively). Age, as a factor of poor prognosis, is mainly directly related to the degree of differentiation of thyroid cancer. The association with age is directly related to tumor differentiation and hence RAl avidity. Nixon et al. found that age and RAl avidity were associated, and younger patients often had higher rates of RAl-avid which was associated with a good outcome (33), while loss of RAl avidity was associated with a poor outcome (35).

The number and location of metastases also affect the prognosis of DTC patients with primary DM. In the present study, the most frequent locus and the number of the brain metastasis was not analyzed. AlDhahri et al. noted that brain metastasis occurs more frequently in the cerebral hemispheres, other sites for intracranial metastasis are the cerebellum, brainstem and pituitary (36). Previous study reported that brain metastases in the brainstem as well as with cranial neuropathy or vision changes could lead to a poor prognosis $(37,38)$. In addition, patients with multiple cranial metastases seemed to had a worse outcome than patients with a single metastasis. Surgical resection of metastatic disease can enhance local disease control and improving quality of life. The NCCN guideline recommended that surgical resection followed by WBRT or SRS plus WBRT was appropriate for patients who had stable systemic disease or were newly diagnosed, while WBRT or SRS was appropriate for patients who had multiple (囚3) metastatic lesions. Therefore, patients with multiply organs 
metastases often had a worse overall or disease specific survival. The study reported by wang et al. found that patients with DM limited to one organ system had a five-year survival of $77.6 \%(p<0.001)$, whilst those who develop second organ involvement by DM had a very poor prognosis with a five-year survival of just $15.3 \%$ (39). This was probably because those patients were not operated due to the high metastatic burden and poor performance status. The Karnofsky Performance Scale (KPS) is an assessment tool for functional impairment. In the American Society for Radiation Oncology evidencebased guideline, KPS was used as a prognostic factor for BM. They defined a KPS score of over 70 points as having a good prognosis. Akiba (40) and Izumi (41) reported that a KPS score over 70 points was a good prognosis factor for metastasis of brain tumor.

This is the first comprehensive study to evaluate the surgery benefit varied by metastatic sites for DTC with distant metastases, but several limitations should be noted. Besides the extent and site of metastatic disease, additional effect modifiers such as surgical resection margins, timing of surgery, type of systemic treatment administered prior to surgery, and coding errors may influence the effect of surgery. We were not able to control these potential modifier effects in this retrospective study due to lack of the information of those variables in the SEER database. Despite propensity score matching used in this study, it is worth nothing that selection bias, such as younger age, better performance status, smaller size of primary tumor, and good response to prior systemic therapy may have influenced the decision to perform surgery, which can affect reliability of this retrospective study. Additionally, the SEER program only included four site-specific distant metastases at the initial diagnosis, and we could not obtain further details concerning the other sites of distant metastases.

\section{Conclusion}

Survival benefit offered by surgery for DTC with primary DM varies by metastatic sites. Local surgery for DTC patients with lung metastasis, liver metastasis, or bone metastasis, whereas the opposite effect is observed among brain metastasis or multiply organs distant metastasis patients. Decisions for primary surgery of DTC patients with primary DM should be tailored according to metastatic site, and mechanisms of first metastasis DTC are needed further understanding.

\section{Declarations}

\section{Competing interests}

The authors declare that they have no competing interests.

Funding

This study was supported by Open Fund of Key Laboratory of Head \& Neck Cancer Translational Research of Zhejiang Province.

Author contributions

Conception or design: WD, ZL, GR. Acquisition, analysis, or interpretation of data: WD, GR, JZ, CT. Drafting of the manuscript: WD. Critical revision of the manuscript for important intellectual content: WD, GR, JZ, 
CT, ZL. Statistical analysis: WD, JZ. Supervision: ZL. All authors have given final approval of the manuscript for submission and publication.

Acknowledgements

No acknowledgements.

Ethics approval and consent to participate

Ethical approval was reviewed and approved by the institutional review board at the Shaoxing Second hospital

Consent for publication

Not applicable.

Availability of data and materials

The datasets used and/or analyzed during the current study are available from the corresponding author on reasonable request.

\section{Abbreviations}

DTC, differentiated thyroid cancer; PTC, papillary thyroid carcinoma, FTC, follicular thyroid carcinoma, DM, distant metastases; RAI: radioactive iodine; EBRT: external beam radiation therapy; WBRT, Whole Brain Radiation Therapy, SRS, stereotatic radiosurgery, OS, overall survival; DSS, disease-specific survival; NIS, sodium iodide symporter, KPS, Karnofsky Performance Scale, HR, hazard ratio; Cl, confidence interval; SEER, Surveillance Epidemiology and End Results, NCl, National Cancer Institute, NCCN, National Comprehensive Cancer Network, NA, not available.

\section{References}

1.

Mihailovic J, Stefanovic L, Malesevic M. Differentiated thyroid carcinoma with distant metastases: probability of survival and its predicting factors. Cancer Biother Radiopharm. 2007 Apr;22(2):250-5. 2.

Shaha AR, Shah JP, Loree TR. Differentiated thyroid cancer presenting initially with distant metastasis. Am J Surg. 1997 Nov;174(5):474-6.

3.

Lang BH, Wong KP, Cheung CY, Wan KY, Lo CY. Evaluating the prognostic factors associated with cancerspecific survival of differentiated thyroid carcinoma presenting with distant metastasis. Ann Surg Oncol. 2013 Apr;20(4):1329-35.

4.

Shoup M, Stojadinovic A, Nissan A, Ghossein RA, Freedman S, Brennan MF, Shah JP, Shaha AR. Prognostic indicators of outcomes in patients with distant metastases from differentiated thyroid 
carcinoma. J Am Coll Surg. 2003 Aug;197(2):191-7.

5.

Mihailovic J, Stefanovic L, Malesevic M, Markoski B. The importance of age over radioiodine avidity as a prognostic factor in differentiated thyroid carcinoma with distant metastases. Thyroid. 2009 Mar;19(3):227-32.

6.

Durante C, Haddy N, Baudin E, Leboulleux S, Hartl D, Travagli JP, Caillou B, Ricard M, Lumbroso JD, De Vathaire F, Schlumberger M. Long-term outcome of 444 patients with distant metastases from papillary and follicular thyroid carcinoma: benefits and limits of radioiodine therapy. J Clin Endocrinol Metab. 2006 Aug;91(8):2892-9.

7.

Lee J, Soh EY. Differentiated thyroid carcinoma presenting with distant metastasis at initial diagnosis clinical outcomes and prognostic factors. Ann Surg. 2010 Jan;251(1):114-9.

8.

Benbassat CA, Mechlis-Frish S, Hirsch D. Clinicopathological characteristics and long-term outcome in patients with distant metastases from differentiated thyroid cancer. World J Surg. 2006 Jun;30(6):108895.

9.

Jonklaas J, Sarlis NJ, Litofsky D, Ain KB, Bigos ST, Brierley JD, Cooper DS, Haugen BR, Ladenson PW, Magner J, Robbins J, Ross DS, Skarulis M, Maxon HR, Sherman SI. Outcomes of patients with differentiated thyroid carcinoma following initial therapy. Thyroid. 2006 Dec;16(12):1229-42. 10.

Huang IC, Chou FF, Liu RT, Tung SC, Chen JF, Kuo MC, Hsieh CJ, Wang PW. Long-term outcomes of distant metastasis from differentiated thyroid carcinoma. Clin Endocrinol (Oxf). 2012 Mar;76(3):439-47. 11.

Haq M, Harmer C. Differentiated thyroid carcinoma with distant metastases at presentation: prognostic factors and outcome. Clin Endocrinol (0xf). 2005 Jul;63(1):87-93.

12.

Sugitani I, Fujimoto Y, Yamamoto N. Papillary thyroid carcinoma with distant metastases: survival predictors and the importance of local control. Surgery. 2008 Jan;143(1):35-42.

13.

Fritz APC, Jack A. International classification of diseases for Oncology. Geneva: World Health Organization; 2000.

14.

Lim H, Devesa SS, Sosa JA, Check D, Kitahara CM. Trends in Thyroid Cancer Incidence and Mortality in the United States, 1974-2013. JAMA. 2017 Apr 4;317(13):1338-1348.

15 .

Yang CQ, Gardiner L, Wang H, Hueman MT, Chen D. Creating Prognostic Systems for Well-Differentiated Thyroid Cancer Using Machine Learning. Front Endocrinol (Lausanne). 2019 May;8:10:288.

16. 
Kang W, Yang S, Zhu-Yue L, Ye-Lei X, Jie L, Xiang Z, Hong-Yuan L. Metastatic Pattern Discriminates Survival Benefit of Primary Surgery for De Novo Stage IV Breast Cancer: A Real-World Observational Study. Eur J Surg Oncol. 2019 Aug;45(8):1364-72.

17.

Sagara Y, Freedman RA, Vaz-Luis I, Mallory MA, Wong SM, Aydogan F, DeSantis S, Barry WT, Golshan M, Patient Prognostic Score and Associations With Survival Improvement Offered by Radiotherapy After Breast-Conserving Surgery for Ductal Carcinoma In Situ: A Population-Based Longitudinal Cohort Study. J Clin Oncol. 2016 Apr 10;34(11):1190-6.

18.

Stürmer T, Rothman KJ, Avorn J, Glynn RJ, Treatment effects in the presence of unmeasured confounding: dealing with observations in the tails of the propensity score distribution-a simulation study. Am J Epidemiol. 2010 Oct 1;172(7):843 - 54.

19.

Rosenbaum PR, Rubin DB. Reducing bias in observational studies using subclassification on the propensity score. J Am Stat Assoc. 1984;79:516-24.

20.

Fine JPGR. A proportional hazards model for the subdistribution of a competing risk. J Am Stat Assoc 1999:496-509.

21.

Walker GV, Giordano SH, Williams M, Jiang J, Niu J, Mackinnon J, Anderson P, Wohler B, Sinclair AH, Boscoe FP, Schymura MJ, Buchholz TA, Smith BD. Muddy water? Variation in reporting receipt of breast cancer radiation therapy by population-based tumor registries. Int J Radiat Oncol Biol Phys. 2013 Jul;15(4):686-93. 86(.

22.

Luster M, Aktolun C, Amendoeira I, Barczyński M, Bible KC, Duntas LH, Elisei R, Handkiewicz-Junak D, Hoffmann M, Jarząb B, Leenhardt L, Musholt TJ, Newbold K, Nixon IJ, Smit J, Sobrinho-Simões M, Sosa JA, Tuttle RM, Verburg FA, Wartofsky L, Führer D, European Perspective on 2015 American Thyroid Association Management Guidelines for Adult Patients with Thyroid Nodules and Differentiated Thyroid Cancer: Proceedings of an Interactive International Symposium. Thyroid. 2019 Jan;29(1):7-26.

23.

Haugen BR, Alexander EK, Bible KC, Doherty GM, Mandel SJ, Nikiforov YE, Pacini F, Randolph GW, Sawka AM, Schlumberger M, Schuff KG, Sherman SI, Sosa JA, Steward DL, Tuttle RM, Wartofsky L. 2015 American Thyroid Association Management Guidelines for Adult Patients with Thyroid Nodules and Differentiated Thyroid Cancer: The American Thyroid Association Guidelines Task Force on Thyroid Nodules and Differentiated Thyroid Cancer. Thyroid. 2016 Jan;26(1):1-133.

24.

Sampson E, Brierley JD, Le LW, Rotstein L, Tsang RW, Clinical management and outcome of papillary and follicular (differentiated) thyroid cancer presenting with distant metastasis at diagnosis. Cancer. 2007 Oct 1;110(7):1451-6.

25.

Page 17/21 
Sugitani I, Fujimoto Y, Yamamoto N. Papillary thyroid carcinoma with distant metastases: survival predictors and the importance of local control. Surgery. 2008 Jan;143(1):35-42.

26.

Haugen BR, Kane MA. Approach to the thyroid cancer patient with extracervical metastases. J Clin Endocrinol Metab. 2010 Mar;95(3):987-93.

27.

Lee HS, Yoo H, Lee SH, Gwak HS, Shin SH. Clinical characteristics and follow-up of intracranial metastases from thyroid cancer. Acta Neurochir (Wien). 2015 Dec;157(12):2185-94. 28.

Saito F, Uruno T, Shibuya H, Kitagawa W, Nagahama M, Sugino K, Ito K. Prognosis After Brain Metastasis from Differentiated Thyroid Carcinoma. World J Surg. 2016 Mar;40(3):574-81.

29.

McWilliams RR, Giannini C, Hay ID, Atkinson JL, Stafford SL, Buckner JC. Management of brain metastases from thyroid carcinoma: a study of 16 pathologically confirmed cases over 25 years. Cancer. 2003 Jul 15;98(2):356-62.

30.

Salvati M, Frati A, Rocchi G, Masciangelo R, Antonaci A, Gagliardi FM, Delfini R. Single brain metastasis from thyroid cancer: report of twelve cases and review of the literature. J Neurooncol. 2001 Jan;51(1):3340.

31.

Misaki T, Iwata M, Kasagi K, Konishi J. Brain metastasis from differentiated thyroid cancer in patients treated with radioiodine for bone and lung lesions. Ann Nucl Med. 2000 Apr;14(2):111-4.

32.

Spitzweg C, Bible KC, Hofbauer LC, Morris JC. Advanced radioiodine-refractory differentiated thyroid cancer: the sodium iodide symporter and other emerging therapeutic targets. Lancet Diabetes Endocrinol. 2014 Oct;2(10):830-42.

33.

Nixon IJ, Whitcher MM, Palmer FL, Tuttle RM, Shaha AR, Shah JP, Patel SG, Ganly I. The impact of distant metastases at presentation on prognosis in patients with differentiated carcinoma of the thyroid gland. Thyroid. 2012 Sep;22(9):884-9.

34.

Brierley J, Tsang R, Panzarella T, Bana N. Prognostic factors and the effect of treatment with radioactive iodine and external beam radiation on patients with differentiated thyroid cancer seen at a single institution over 40 years. Clin Endocrinol (Oxf). 2005 Oct;63(4):418-27.

35.

Riesco-Eizaguirre G, Gutiérrez-Martínez P, García-Cabezas MA, Nistal M, Santisteban P. The oncogene BRAF V600E is associated with a high risk of recurrence and less differentiated papillary thyroid carcinoma due to the impairment of $\mathrm{Na}+/ \mathrm{I}$ - targeting to the membrane. Endocr Relat Cancer. 2006 Mar;13(1):257-69.

36. 
Al-Dhahri SF, Al-Amro AS, Al-Shakwer W, Terkawi AS. Cerebellar mass as a primary presentation of papillary thyroid carcinoma: case report and literature review. Head Neck Oncol. 2009 Jun;29:1:23.

37.

Jakola AS, Gulati S, Nerland US, Solheim O. Surgical resection of brain metastases: the prognostic value of the graded prognostic assessment score. J Neurooncol. 2011 Dec;105(3):573-81.

38.

Villà S, Weber DC, Moretones C, Mañes A, Combescure C, Jové J, Puyalto P, Cuadras P, Bruna J, Verger E, Balañà C, Graus F. Validation of the new Graded Prognostic Assessment scale for brain metastases: a multicenter prospective study. Radiat Oncol. 2011 Mar;2:6:23.

39.

Wang LY, Palmer FL, Nixon IJ, Thomas D, Patel SG, Shaha AR, Shah JP, Tuttle RM, Ganly I. Multi-organ distant metastases confer worse disease-specific survival in differentiated thyroid cancer. Thyroid. 2014 Nov;24(11):1594-9.

40 .

Akiba T, Kunieda E, Kogawa A, Komatsu T, Tamai Y, Ohizumi Y. Re-irradiation for metastatic brain tumors with whole-brain radiotherapy. Jpn J Clin Oncol. 2012 Apr;42(4):264-9.

41.

Izumi J, Watanabe O, Koga M, Anbai A, Suzuki A, Hashimoto M. Stereotactic irradiation for brain metastases: analysis of prognostic factors in survival. Akita J Med. 2011;38:49-55.

\section{Figures}

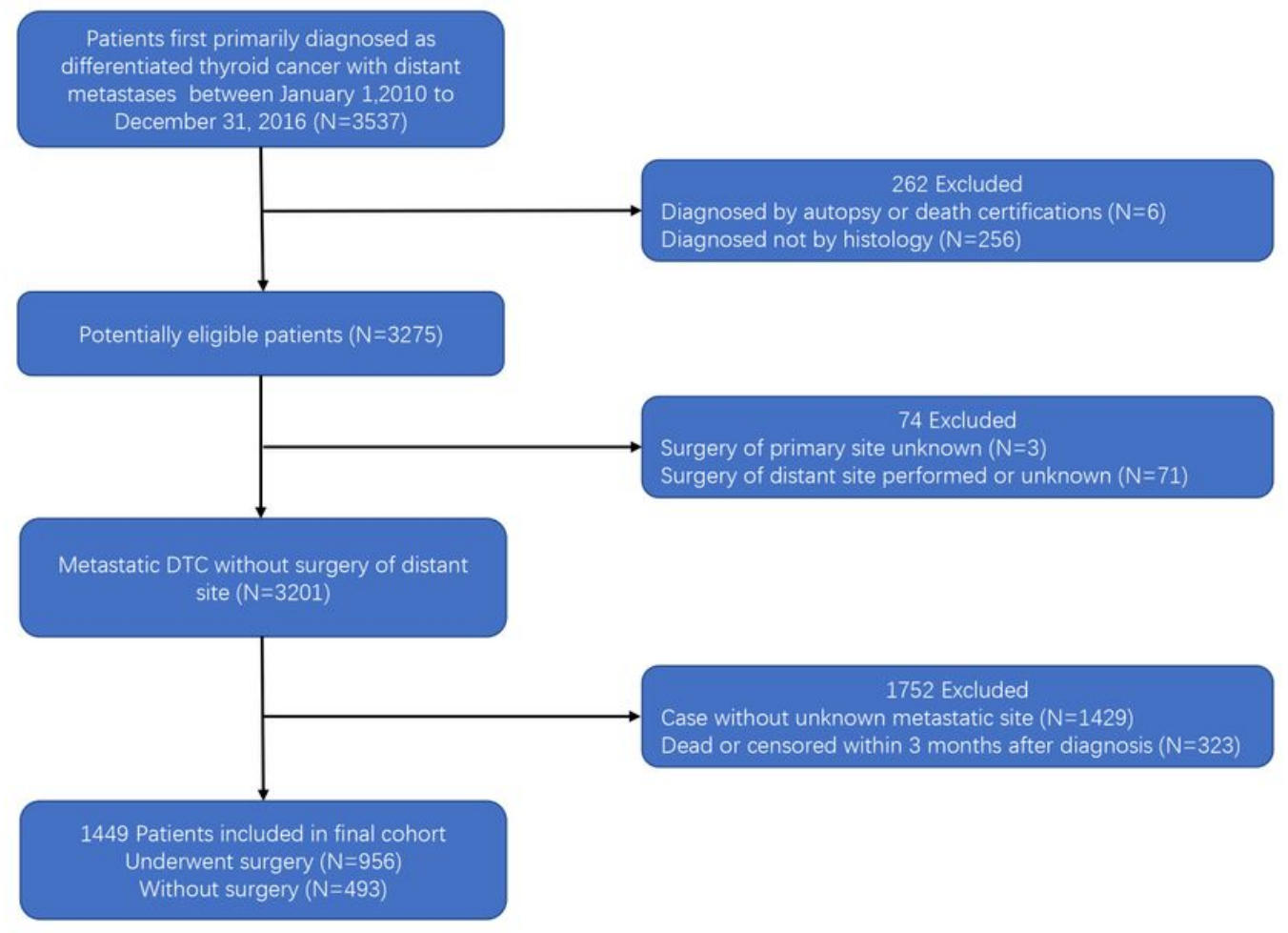


Figure 1

Flow diagram of patient population.

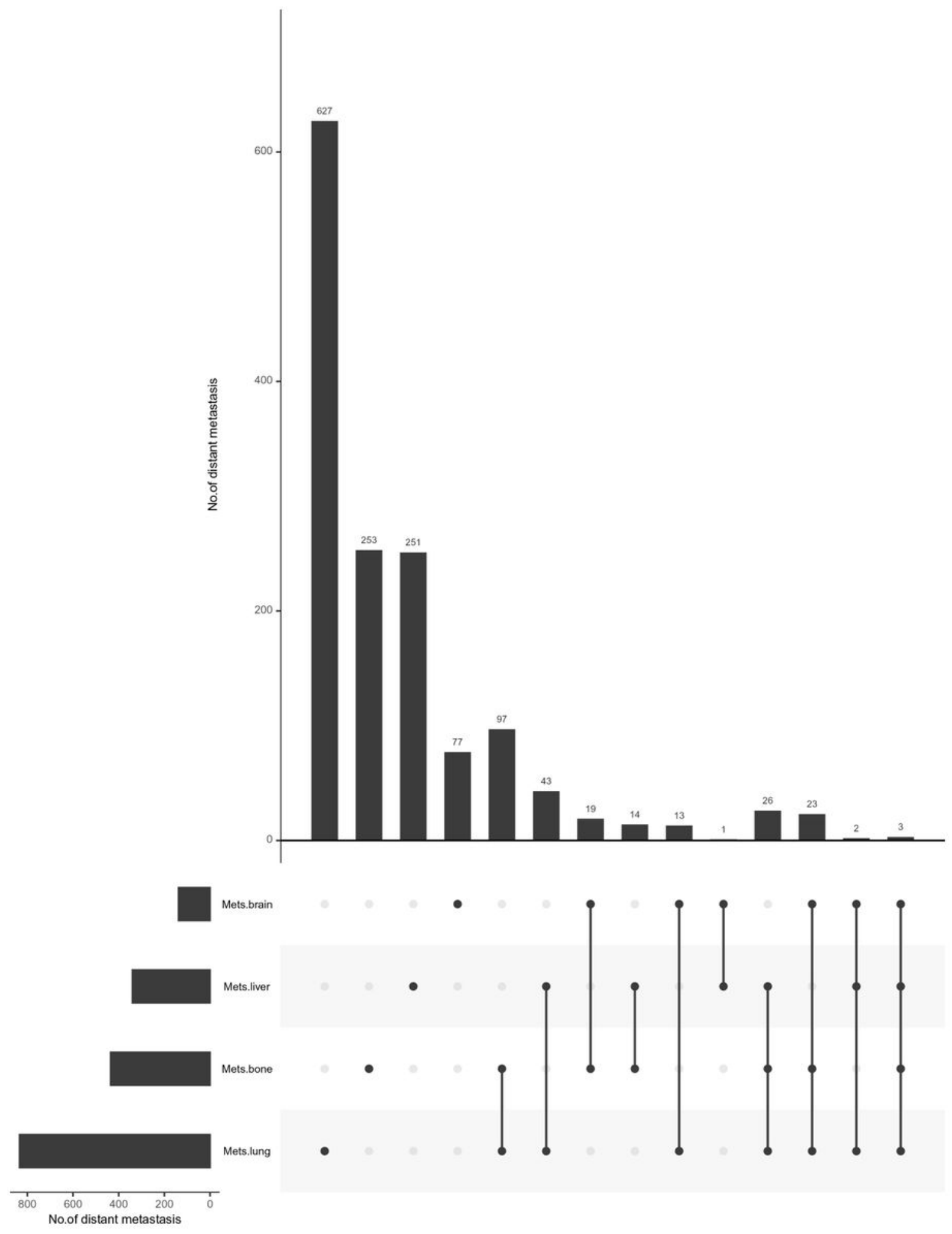

Figure 2

The distribution of metastatic sites in differentiated thyroid cancer patients with distant metastases. 


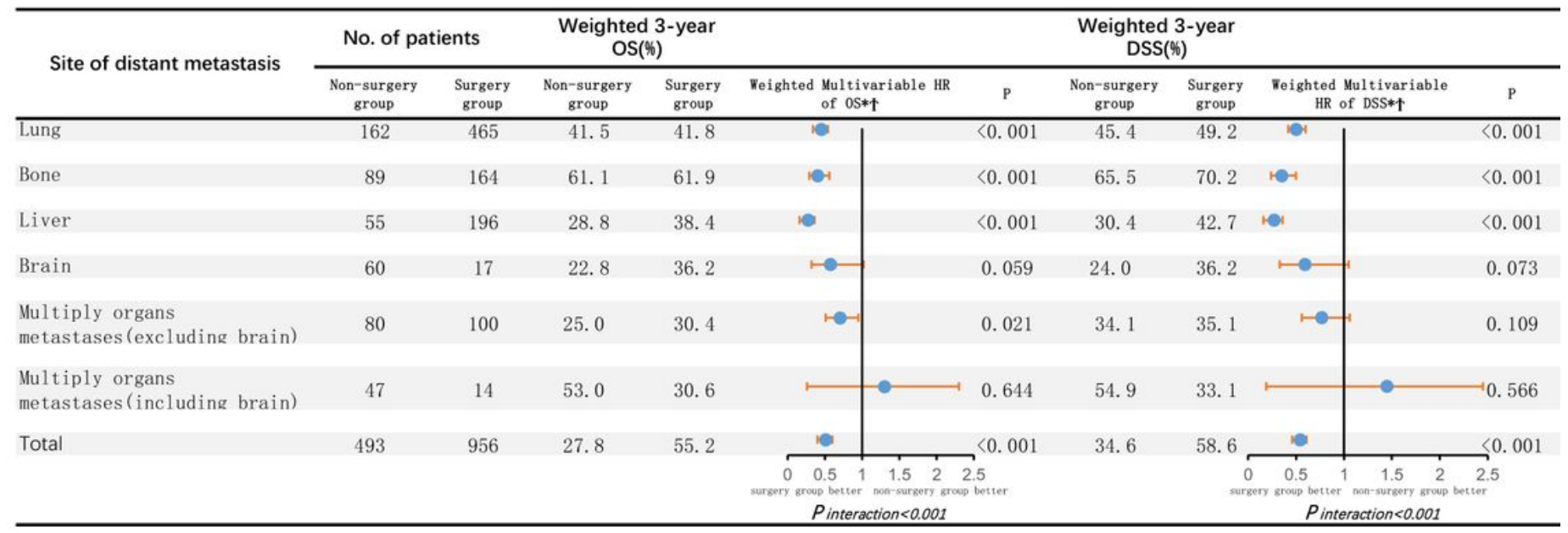

\section{Figure 3}

Hazard ratio comparing OS/DSS between surgery group and non-surgery group according to metastatic site for patients with distant metastases. (*) Weighted by inverse propensity score. ( $($ ) Multivariate analysis adjusted by patient age, year of diagnosis, race, sex, tumor size, regional lymph node status, marital status, distant metastatic sites, histology type, nuclear grade, chemotherapy, radiation therapy. Abbreviations: OS, overall survival; DSS, disease specific survival; HR, hazard ratio. 